\title{
Comment on
}

\section{"Significance of "high probability/low damage" versus "low probability/high damage" flood events" by Merz et al. (2009)}

\author{
C. M. Rheinberger \\ WSL Institute for Snow and Avalanche Research SLF, Risk Management Group, 7260 Davos, Switzerland
}

Received: 14 September 2009 - Accepted: 29 November 2009 - Published: 5 January 2010

\begin{abstract}
This comment is meant to shed some light on the use of so-called "risk aversion functions" in the management of flood risks and other natural hazards as recently proposed in this journal (Merz et al., 2009). In particular, I resume the discussion as to whether the relative damage is a suitable indicator of risk aversion and lay out why the use of this indicator may lead to inefficient decisions upon flood mitigation measures.
\end{abstract}

\section{Introduction}

In a recent paper, Merz et al. (2009, hereafter referred to as MET) analyze the contribution of "low probability/high damage" flood events to the overall expected annual flood damage (EAFD). Assuming that such "low probability/high damage" events are perceived worse by society than it is expressed by their share of the EAFD, MET suggest to penalize events with disastrous consequences in the appraisals of mitigation options by integrating risk aversion into decision making. In particular, MET propose the use of a simple power function to weight the EAFD at a particular flood site relative to the values at risk within this site. This weighting procedure is illustrated by means of three case studies from German flood sites based on which MET conclude that the inclusion of risk aversion into the risk appraisal may help to overcome the gap between flood risk as perceived by society and flood risk as calculated by flood risk engineers.

I congratulate MET on their paper, which is a valuable contribution to the field of flood risk management. Yet, I wish to raise some doubts about the adequacy of using the relative damage as an indicator of risk aversion. I even dare to say that this indicator inevitably leads to decisions about

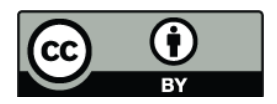

Correspondence to: C. M. Rheinberger (rheinberger@slf.ch) mitigation options, which are far from socially optimal. I am going to back this claim by the following example. Suppose there are two flood sites A and B; site A may be in a city of one million inhabitants (e.g. Cologne) and site B in a town of 20000 inhabitants (e.g. Doebeln). Now, consider the damaging flood events on which MET report. At both sites, damages of roughly 80 million $€$ were to be observed during the last flood events. What would have been the consequence of assessing these two events by the proposed weighting scheme?

To answer this question I apply the most conservative risk aversion function proposed by MET in the left panel of their Fig. 1:

$\alpha\left(D_{\mathrm{j}}\right)=\left\{\begin{array}{lll}1 & \text { if } & D_{\mathrm{j}} / \mathrm{VaR} \leq 1 \%, \\ \frac{2000}{19} \cdot \frac{D_{\mathrm{j}}}{\mathrm{VaR}} & \text { if } & 1 \%<D_{\mathrm{j}} / \mathrm{VaR} \leq 20 \%, \\ 10 & \text { if } & D_{\mathrm{j}} / \mathrm{VaR}>20 \% .\end{array}\right.$

where $\alpha\left(D_{\mathrm{j}}\right)$ is the risk aversion factor in dependence on the expected damage $D_{\mathrm{j}}$ of flood event $\mathrm{j}$, and VaR denotes the absolute value at risk. Figure 1a compares the weighted damages for sites A and B using Eq. (1) to determine the risk aversion factor. For ease of calculation, I assume that 80 million $€$ correspond to a relative damage of $0.1 \%$ at site A and of 5\% at site B (based on the simplifying assumption that the values at risk grow linearly with population size). It is easy to see that the weighted damage at site B is about five times larger than the weighted damage at site $\mathrm{A}$ although the expected damage is in both cases 80 million $€$. The difference between the weighted damages becomes even more drastically if one considers a maximum probable loss event in which $25 \%$ of site B (and $0.5 \%$ of site A) would be damaged, i.e. one expects a maximum loss of 400 million $€$ (Fig. 1b).

Thus, if one would apply this weighted damage to calculate the "societal perceived risk" as proposed by Eq. (7) in the MET paper, this risk would be five times larger for site B

Published by Copernicus Publications on behalf of the European Geosciences Union. 

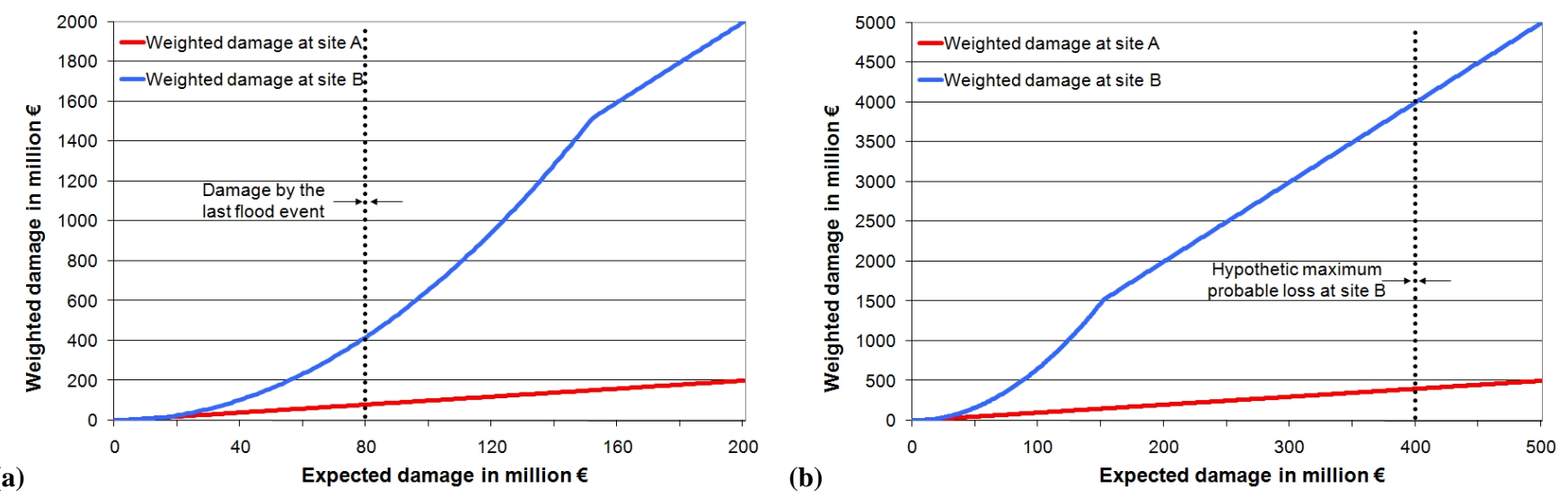

Fig. 1. Weighted versus expected damage for two flood sites with varying VaR. Panel (a) focuses on a damage size of 80 million $€$ which has been observed in past flood events in Germany; panel (b) focuses on a hypothetic maximum probable loss of 400 million $€$.

as for site A (assuming that both flood events are equally probable). In other words, avoiding a loss of 80 million $€$ at site $\mathrm{B}$ would be valued five times as worthy as avoiding the same loss at site A, although a loss of 80 million $€$ at site $A$ is likely to have a shorter return period simply because the exceedance probability of a relative damage of $0.1 \%$ is larger than the one of a relative damage of $5 \%$.

What has gone wrong in this risk appraisal? Obviously, relating the weighting function to the respective values at risk results in two different disutility functions. However, under expected utility theory, threats are not evaluated relative to a reference point and background information on the size of the affected flood site should not influence the decision maker's preferences for avoiding a particular loss. I conclude that, while the overweighting of "low probability/high damage" events might be justified by normative considerations, the use of the relative damage as an indicator for determining the risk aversion factor is neither theoretically warranted nor does it lead to optimal mitigation decisions.
Let me be clear about this. I do not think that scale differences should be neglected in flood risk management. Admittedly, a flood loss of 80 million $€$ could cause larger indirect costs when occurring in a small town as when occurring in a large city. However, economic theory suggests to directly estimate these additional indirect costs instead of incorporating them into a weighting scheme that is doubtful at best.

Acknowledgements. Stimulating discussions with Michael Bründl are acknowledged. The usual disclaimer applies.

Edited by: F. Guzzetti

Reviewed by: B. Merz and another anonymous referee

\section{References}

Merz, B., Elmer, F., and Thieken, A. H.: Significance of "high probability/low damage" versus "low probability/high damage" flood events, Nat. Hazards Earth Syst. Sci., 9, 1033-1046, 2009, http://www.nat-hazards-earth-syst-sci.net/9/1033/2009/. 